\title{
Performance of HARQ in Device-to-Device Communication
}

\author{
Wenji Feng, Yafeng Wang, Lei Yang \\ Wireless Theory \& Technology lab (WT\&T), Beijing University of Posts and Telecommunications, Beijing, China \\ fwj10171017@sina.com,wangyf@bupt.edu.cn \\ Email: fwj10171017@sina.com, wangyf@bupt.edu.cn,yanglei5658@gmail.com
}

Received July, 2013

\begin{abstract}
In this paper, we study D2D (Device-to-Device) communication underlying LTE-Advanced uplink system. Since D2D communication reuses uplink resources with cellular communication in this scenario, it's hard for D2D users to avoid the interference from cellular users while cellular users are communication with eNB (evolved Node B). HARQ (Hybrid Automatic Repeat reQuest) is widely used in LTE-Advanced system in order to improve the accurate rate of cellular communication. Hence, we consider studying the integration of D2D with HARQ, so as to achieve the purpose of improving the throughput of D2D communication and the performance of overall system. Synchronous HARQ is considered to introduce into D2D communication procedures. What's more, this idea will be taken into system-level simulation. From the simulation results, we can see that the throughput of D2D communication gets a lot of gain and the performance of overall system is improved as well. In addition, Synchronous HARQ technique can significantly decrease the BLER (Block Error Rate) of D2D communication, especially for which in a bad channel condition.
\end{abstract}

Keywords: D2D Communication; Synchronous HARQ; LTE-Advanced System; Uplink; BLER

\section{Introduction}

3GPP Long Term Evolution (LTE) technology has been proved to have outstanding performance, especially in the measures of spectral efficiency and throughput, cell edge and peak values in a cellular, frequency reuse one network $[1,2]$. Hence, major efforts have been spent on the development of LTE. Currently the further evolu-tion of such systems has been started under the scope of IMT-Advanced [3-5].

The device-to-device communication (D2D) technol-ogy, also known as proximity-based services (ProSe), is introduced to the LTE-Advanced system [6-7]. However, the D2D communication is sharing authorized frequency band with cellular communication by the way of or-thogonal method or multiplexing method. The introduc-tion of D2D communication is to improve the throughput of the whole cellular system. It contributes to higher sys-tem spectrum efficiency as well. When D2D users reuse cellular frequency resources, it is hard to avoid the inter-ference from other cellular users in the same cell. It may affect the performance of D2D communication to some extent.

Hybrid automatic repeat request technology (HARQ) is widely used in LTE-Advanced. HARQ can be divided into synchronous HARQ and asynchronous HARQ in accordance with the time of occurrence of the re- trans-mission [8]. Synchronous HARQ means the transmission of a HARQ process (retransmission) occurs at a fixed moment. Asynchronous HARQ refers to a retransmission that the HARQ process can occur at any time. Synchro-nous HARQ is considered to introduce into D2D com-munication procedures.

This paper focuses on studying the integration of D2D with synchronous HARQ, and analysis the overall system performance after joining the mechanism.

This paper is organized as follows: Section 2 describes the simulation platform. Section 3 compares and analysis the overall system performance with or without D2D communication. Finally concluding remarks are made in Section 4.

\section{Procedures and Simulation Platform}

We usually use HARQ to guarantee the accuracy of data communication in LTE-Advanced system. Thus, we as well consider adding HARQ into D2D communication to guarantee the accuracy, and improve the performance of overall system.

D2D communication reuses uplink resources of LTE-Advanced in our simulation. It means that D2D users only communicate during uplink slot. Figure 1 shows the procedures of the integration of D2D with HARQ. 


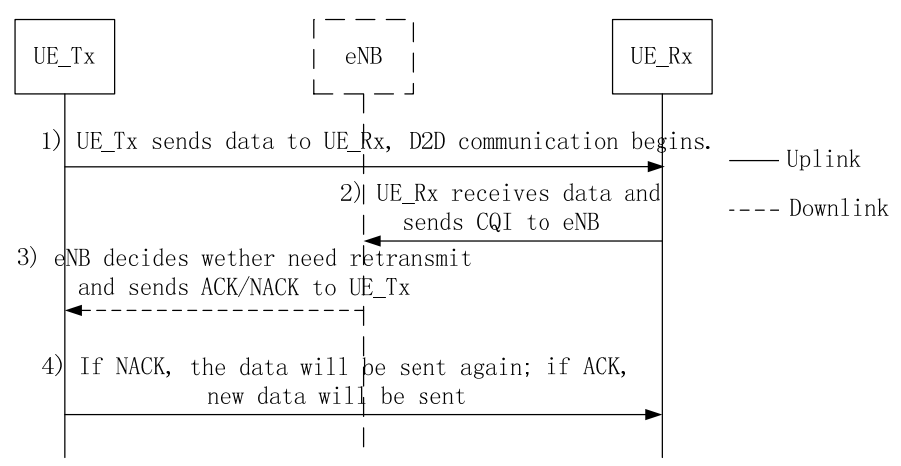

Figure 1. Flow chart of synchronous HARQ for D2D.

Where CQI denotes Channel Quality Indicator, ACK denotes Acknowledgement, and NACK denotes Negative Acknowledgement.

Synchronous HARQ is introduced into simulation platform. Different HARQ process ID is recorded by system. In the same process, when the transmission occurs, eNB merges SINR (Signal-to-Interference plus Noise Ratio), and then determine whether to retransmit. If there is no SINR data in the process, eNB can use current SINR without merging. After deciding whether needs to retransmit, there will be two branches:

1) Retransmit:

a) If system calls retransmission, the number of retransmission (ReTxNUM) will be recorded. The current SINR, MCS and RB (Resource Block) ID will be recorded as well, which are merging data for next retransmission.

b) If system calls retransmission, and current ReTxNUM is greater than Max-ReTxNUM, clear all data of current process. System gives up transmitting this packet.

2) Non retransmit:

If the packet is successful transmitted, system clear all data of current process.

The flow chart of synchronous HARQ for D2D simulation platform is given in Figure 2.

The generation of retransmission probability in system-level simulation is based on the mapping relationship of SINR and BLER. Also the MCS and the size of $\mathrm{RB}$ will affect this mapping relationship. In our HARQ process the MCS is set to 28, and the size of RB is 10 . Table 1 shows the mapping relationship of SINR and BLER.

The simulation platform consists of 7 base stations (21 sectors). The radius of each cell is $500 \mathrm{~m}$. The distance between transmitter and receiver is $10 \mathrm{~m}$ to $20 \mathrm{~m}$. D2D communication reuses UL resources of LTE-Advanced, which contains $46 \mathrm{RB}$. The transmission power of D2D transmitter is $20 \mathrm{dBm}[9,10]$. The system bandwidth is 10 $\mathrm{MHz}$ and the carrier frequency is $2 \mathrm{GHz}$. We distribute 210 cellular UEs into overall system so that there are 8 to 10 cellular UEs in each cell. Also we distribute one pair

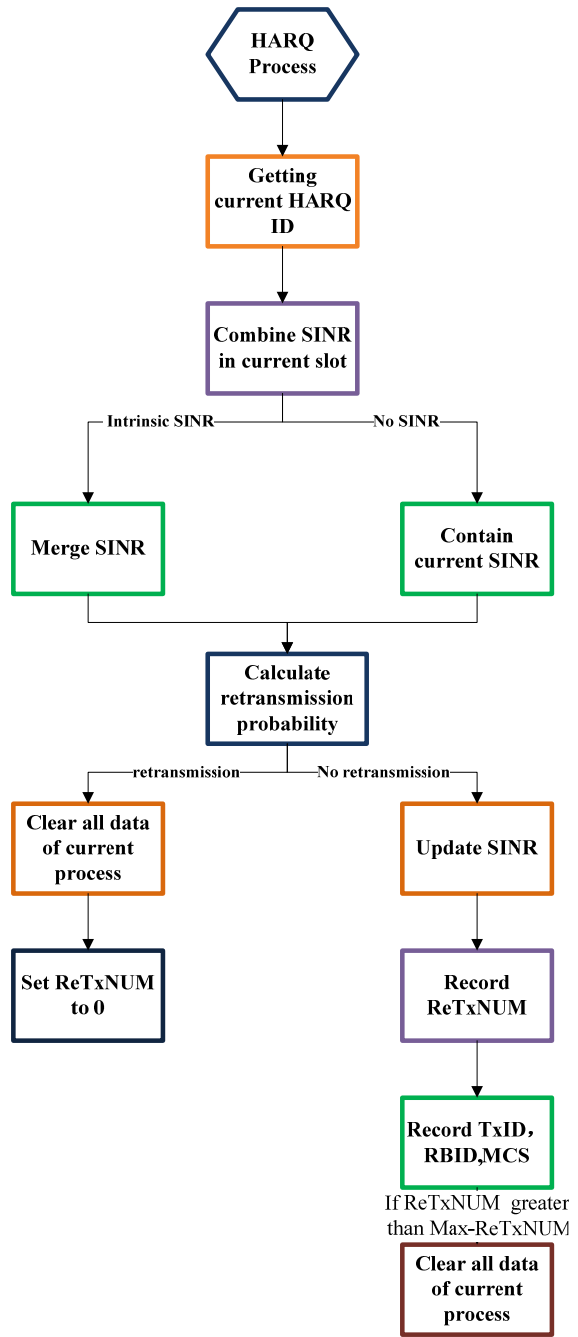

Figure 2. Flow chart of synchronous HARQ for D2D.

of D2D UEs into one cell amount to 21 pairs of D2D UEs. The permanent MCS of D2D communication is 28. The path loss model and corresponding shadow fading model of D2D communication are referred as model of Urban Macro(UMa) in [11], where it can be further categorized as Line-of-sight (LOS) and Non-line-of-sight (NLOS). 
Table 1. Mapping relationship of SINR and BLER.

\begin{tabular}{cc}
\hline \multicolumn{2}{c}{ MCS=28 } \\
SIZE OF RB=10 \\
\hline SINR & BLER \\
\hline 17.2 & 1 \\
17.4 & 0.96 \\
17.6 & 0.864271 \\
17.8 & 0.41018 \\
18 & 0.103235 \\
18.2 & 0.020804 \\
18.4 & 0.00529 \\
\hline
\end{tabular}

LOS:

$P L=16.9 \log _{10}(d[m])+46.8+20 \log _{10}\left(f_{c}[G H z] / 5.0\right)(1)$

NLOS:

$P L=40 \log _{10}(d[k m])+30 \log _{10}\left(f_{c}[M H z]\right)+49$

The probability of LOS is as follow:

$$
P_{L O S}=\left\{\begin{array}{cc}
1, & d \leq 4 \\
\exp (-(d-4) / 3), & 4<d<60 \\
0, & d \geq 60
\end{array}\right.
$$

Where $P L$ denotes the path loss, $d$ is the distance between D2D users, $f_{c}$ is carrier frequency, and $P_{L O S}$ is the probability of LOS.

The parameters of simulation are listed in Table 2.

\section{Results and Analyses}

Due to the introduction of synchronous HARQ for D2D communication, the overall system throughput may have been affected. We consider two different simulation scenarios:

a) Case BASIC:

This case will be the one that there is no HARQ in D2D communication. This is a case used as the standard of comparison system performance.

b) Case HARQ:

This case will be the one that joining HARQ into D2D communication.

Both of these two simulation scenarios set the D2D transmission power to $20 \mathrm{dBm}$. The scheduling mode of $\mathrm{D} 2 \mathrm{D}$ is the same and D2D communication reuse $10 \mathrm{RB}$ of cellular communication.

Figure 3 shows the results of two simulation cases.

As Figure 3 shows, the eNB throughput of both cases is the same. It means that the integration of D2D with HARQ does not affect the average throughput of eNB. Since both cases' throughput of cell-edge users are the same as well, we can draw a conclusion that HARQ for D2D communication does not affect the average throughput either. The reasons are as follows:
In our simulation, the interference against eNB and cellular UE is associated with D2D communication. As is in both Case BASIC and Case HARQ, the interference from D2D communication is just the same, the results of eNB throughput and cell edge user throughput turn out to be the same.

Figure 3 also shows that the integration of D2D with HARQ increases 5.6\% D2D communication throughput gain. What's more, the overall system gets $1.9 \%$ throughput gain. In view of this, after joining the HARQ into D2D communication, the performance of D2D communication and overall system has certain promotion. In addition, BLER of case HARQ is $20 \%$ smaller than that of case BASIC. Thus it can be seen that the accuracy of D2D communication gets enhanced after using HARQ.

Table 2. List of simulation parameters.

\begin{tabular}{ll}
\hline Parameters & Value \\
\hline Number of eNB & 7 base stations (21 sectors) \\
Radius of cell & $500 \mathrm{~m}$ \\
Distance of D2D & $10 \mathrm{~m}$ to $20 \mathrm{~m}$ \\
communication & Uplink/D2D and cellular \\
& communication reuse 46 RB \\
System resources & $20 \mathrm{dBm}$ \\
Transmission power of D2D & $10 \mathrm{MHz}$ \\
System bandwidth & $2 \mathrm{GHz}$ \\
Carrier frequency & 210 \\
Number of cellular UE & 21 \\
Number of D2D pairs & 8 to 10 cellular UEs in each cell; \\
& one pair of D2D-UE into one cell \\
Distribution & 28 \\
& $-174 \mathrm{dBm} / \mathrm{Hz}$ \\
MCS of D2D & Proportional Fair \\
Thermal noise density & \\
\hline
\end{tabular}

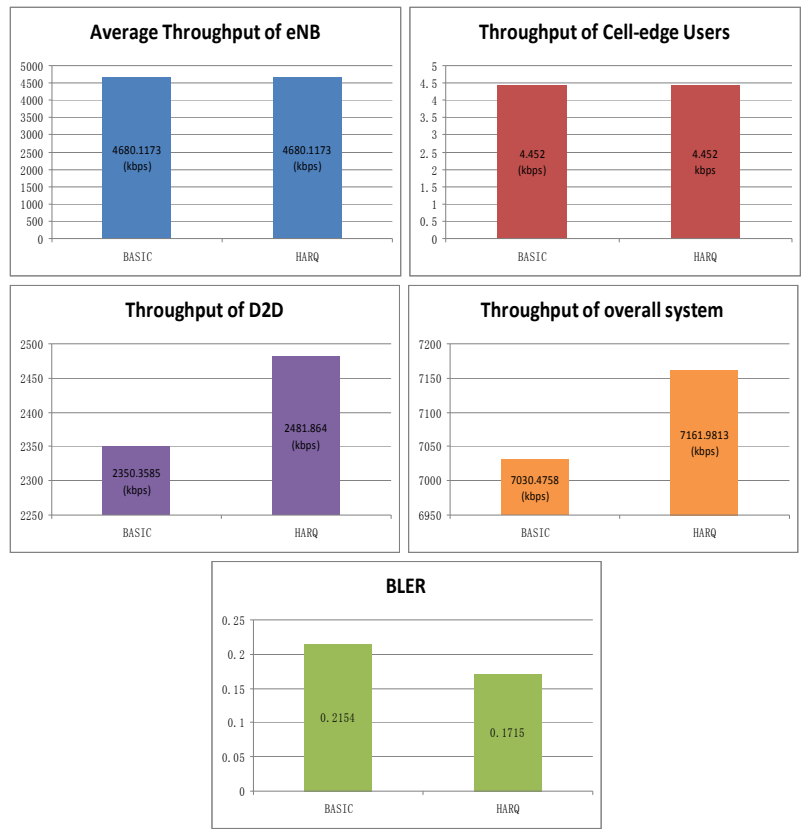

Figure 3. Results of two simulation cases. 
Figure 4 refers to the throughput CDF curves of both case BASIC and case HARQ.

In Figure 4, the performance of D2D communication throughput, which enables HARQ technique, is better than the one without HARQ technique. The D2D UEs, whose throughput is less than $1000 \mathrm{kbps}$, of case HARQ are obviously less than those of case BASIC. It shows that HARQ technique can increase the throughput of low-throughput D2D UEs.

Since all parameters in both Case BASIC and Case HARQ are the same, we consider comparing the throughput of each D2D UE from different cases. Figure 5 shows the throughput difference between Case BASIC and Case HARQ.

From Figure 5 we can find that after using HARQ about 50\% D2D UEs do not get any throughput gain, because we set the transmission power to $20 \mathrm{dBm}$, which is the maximal power for UE. At the same time, about $40 \%$ D2D UEs increase 0 to 500 kbps throughput gain. What's more, 10\% D2D UEs after using HARQ get 500 to $1200 \mathrm{kbps}$ throughput gain. We can assume that if lower transmission power is set for D2D UEs, which means D2D communication get lower SINR in the same channel condition, the gain from HARQ will be greater.

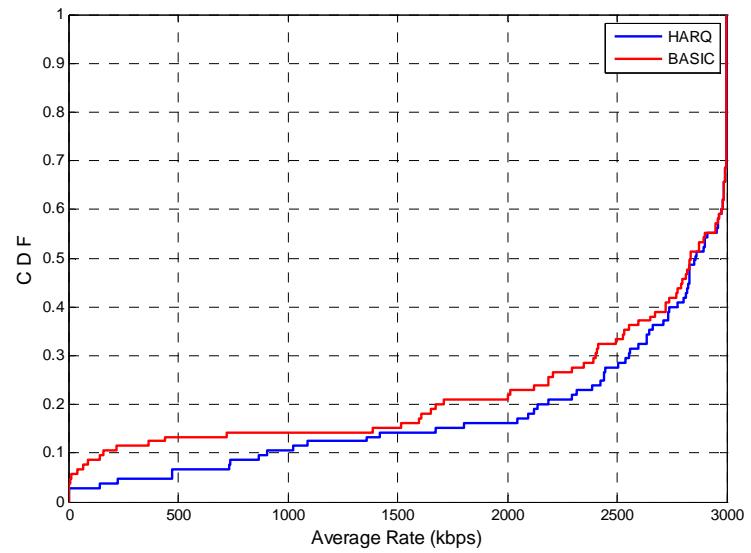

Figure 4. The throughput CDF curves.

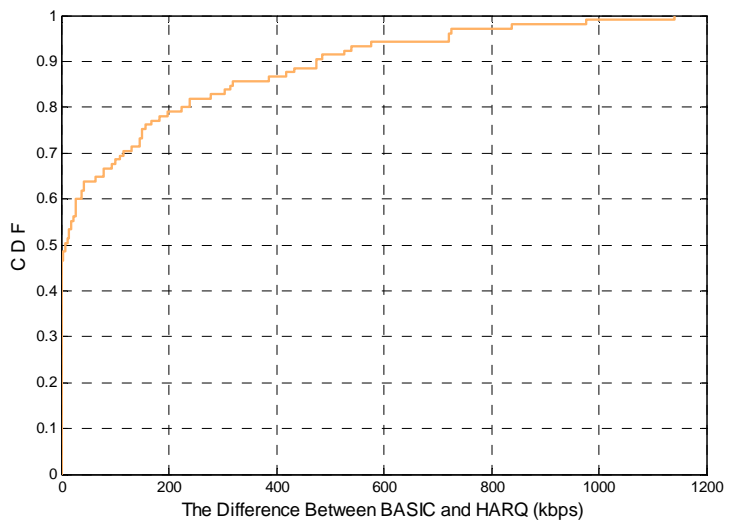

Figure 5. Throughput difference CDF curves.

\section{Conclusions}

In this paper we introduce synchronous HARQ into D2D communication, and we have Case BASIC and Case HARQ for simulation. Case BASIC is the case used as the standard of comparison system performance. In the simulation of Case HARQ, the throughput of eNB and cellular communication is not affected. At the same time, the throughput of D2D communication gets a lot of gain. Due to the gain of D2D communication, the throughput of overall system gets gain as well. What's more, HARQ technique can improve the accurate rate of D2D communication, especially for which in a bad channel condition. Hence, the introduction of HARQ for D2D communication is viable and necessary. For further study, we should further optimize HARQ of D2D communication, so that we can make the overall system gain maximization.

\section{Acknowledgements}

This paper is supported by National Key Technology R\&D Program of China under grant No. 2012ZX03003011.

\section{REFERENCES}

[1] H. Ekstrom, A. Furusk"ar, J. Karlsson, M. Meyer, S. Parkvall, J. Torsner and M. Wahlqvist, "Solutions for the 3G Long-term Evolution,” IEEE Communications Magazine, Vol. 44, No. 3, 2006, pp. 2432-2455. doi:10.1109/MCOM.2006.1607864

[2] ITU, "ITU-R; Recommendation M. 1645 Framework and Overall Objectives of the Future Development of IMT-2000 and Systems Beyondimt-2000,” 2003.

[3] "Estimated Spectrum Bandwidth Requirements for the Future Development of IMT-2000 and IMT-Advanced," ITU.

[4] D. C. Lee and Y. H. Kwon, "Performance Benefits of Uplink Packet Relay Protocols for Cellular-like Systems: Quantitative Analysis," IEEE Transactions Wireless Communications, Vol. 5, No. 7, 2006, pp. 1569-1574. doi:10.1109/TWC.2006.1673062

[5] S. Haykin, "Cognitive Radio: Brain-empowered Wireless Communications," IEEE Journal on Selected Areas in Communications, Vol. 23, No. 2, 2005, pp. 201-220. doi:10.1109/JSAC.2004.839380

[6] H.-Y. Hsieh and R. Sivakumar, "On Using Peer-to-peer Communication in Cellular Wireless Data Networks," IEEE Transactions on Mobile Computing, Vol. 3, No. 1, 2004, pp. 57-72. doi:10.1109/TMC.2004.1261817

[7] P. J“anis, Y. Chia-Hao, K. Doppler, C. Ribeiro, C. Wijting, K. Hugl, O. Tirkkonen and V. Koivunen, "Device-to-device Communication Underlaying Cellular Communications Systems," Submitted to: International Journal of Communications, Network and System Sciences (IJCNS).

[8] D. J. Dechene and A. Shami, "Energy Efficient Resource 
Allocation in Sc-Fdma Uplink with Synchronous Harq Constraints," in Communications (ICC), 2011 IEEE International Conference on, 2011, pp. 1-5.

[9] V. Chandrasekhar and Z. Shen, “Optimal Uplink Power Control in Twocell Systems with Rise-over-thermal Constraints," IEEE communications letters, Vol. 12, No. 3, 2008, pp. 173-175. doi:10.1109/LCOMM.2008.071697

[10] H. M. Wang and D. J. Jiang, “A Novel Bidirectional Re- source Allocation to Decrease Signaling for Retransmission in LTE System," in Vehicular Technology Conference, 2008. VTC Spring 2008. IEEE, 2008), pp. 2269-2271.

[11] Rep. ITU-R M.2135, “Guidelines for Evaluation of Radio Interface Technologies for IMT-Advanced,” Revision 1 to Document 5D/TEMP/90-E, 1st July 2008. 